\title{
Evidence of chemical exchange in recombinant Major Urinary Protein and quenching thereof upon pheromone binding
}

\author{
Chiara Perazzolo • Mariachiara Verde • \\ Steve W. Homans · Geoffrey Bodenhausen
}

Received: 26 June 2006/ Accepted: 30 October 2006/ Published online: 7 March 2007

(C) Springer Science+Business Media B.V. 2007

\begin{abstract}
The internal dynamics of recombinant Major Urinary Protein (rMUP) have been investigated by monitoring transverse nitrogen-15 relaxation using multiple-echo Carr-Purcell-Meiboom-Gill (CPMG) experiments. While the ligand-free protein (APOrMUP) features extensive evidence of motions on the milliseconds time scale, the complex with 2-methoxy-3isobutylpyrazine (HOLO-rMUP) appears to be much less mobile on this time scale. At $308 \mathrm{~K}$, exchange rates $k_{\mathrm{ex}}=500-2000 \mathrm{~s}^{-1}$ were typically observed in APOrMUP for residues located adjacent to a $\beta$-turn comprising residues $83-87$. These residues occlude an entry to the binding pocket and have been proposed to be a portal for ligand entry in other members of the lipocalin family, such as the retinol binding protein and the human fatty-acid binding protein. Exchange rates and populations are largely uncorrelated, suggesting local
\end{abstract}

Electronic supplementary material The online version of this article (doi:10.1007/s10858-006-9110-1) contains supplementary material, which is available to authorized users.

C. Perazzolo $(\bowtie) \cdot$ M. Verde $\cdot$ G. Bodenhausen

Institut des Sciences et Ingénierie Chimiques,

Ecole Polytechnique Fédérale de Lausanne,

1015 Lausanne, Switzerland

e-mail: Chiara.Perazzolo@epfl.ch

S. W. Homans

Institute of Molecular and Cellular Biology,

University of Leeds, Woodhouse Lane, LS2 9JT Leeds, UK

G. Bodenhausen

Département de chimie, associé au CNRS,

École Normale Supérieure, 24 rue Lhomond,

75231 Paris Cedex 05, France 'breathing' motions rather than a concerted global conformational change.

Keywords Carr-Purcell-Meiboom-Gill (CPMG) . Slow protein dynamics - Recombinant Major Urinary Protein (rMUP) · 2-Methoxy-3-isobutylpyrazine (IBMP)

\section{Introduction}

A good grasp of internal dynamics is of crucial importance to understand the biological role and the properties of proteins. NMR spectroscopy offers the unique ability to monitor dynamics over a wide range of time scales. Fast motions (picoseconds to nanoseconds) can be detected using the model-free approach (Lipari and Szabo 1982a, b), while a range of other techniques are used to detect alternative time scales (Carr and Purcell 1954; Meiboom and Gill 1958; Deverell et al. 1970; Wagner et al. 1985; Kloiber and Konrat 2000; Frueh 2002; Früh et al. 2002; Wist et al. 2004). In particular, chemical or conformational processes that stochastically change the magnetic environment of a spin and occur on a time scale of microto milliseconds are known as 'chemical exchange'. Such slow processes can be studied by NMR using Carr-Purcell-Meiboom-Gill experiments (CPMG) (Carr and Purcell 1954; Meiboom and Gill 1958; Loria et al. 1999; Millet et al. 2000; Mulder et al. 2001a; Wang and Palmer 2003; Ishima and Torchia 2005). Note that 'slow processes' in this context should not be confused with 'slow exchange' in the sense established by Millet (when $p_{\mathrm{a}} » p_{\mathrm{b}}$ ), who used this expression whenever the parameter $\alpha=\left(\delta R_{\mathrm{ex}} / R_{\mathrm{ex}}\right) /\left(\delta B_{0} / B_{0}\right)$ which 
can range between $0 \leq \alpha \leq 2$, is smaller than one (Millet et al. 2000). Many critical biological activities occur in this micro- to millisecond time scale: for example many ligand-binding on- and off-rates (Mulder et al. 2001b, c, Akke 2002 and bibliography therein).

MUP proteins are part of the lipocalin family and are produced mainly by sexually mature male mice. MUPs can bind a variety of volatile pheromones that have important effects on the mouse physiology and behaviour, like acceleration of female puberty, blocking of pregnancy and inter-male aggression. The main function of MUPs is to act as a slow releaser, allowing the chemical signal to persist longer in the environment. Their 3D fold comprises an eightstranded $\beta$-barrel and a long $\alpha$-helix, so that the interior of the barrel forms a hydrophobic cavity where the binding site is located. The general name for an isoform of the family of Major Urinary Proteins is recombinant MUP (rMUP) (Ferrari et al. 1997; Lücke et al. 1999), and our isoform is constituted of 162 amino-acids, with a mass around $20 \mathrm{kDa}$, and so it is small enough to be analysed readily by high resolution NMR. Fast motions on time-scales between pico- and nano-seconds have been studied in both APO-rMUP and in the HOLO-rMUP complex with the pheromone 2-methoxy-3 isobutylpyrazine (Bingham et al. 2004).

The aim of the present work is to characterize conformational exchange processes taking place on micro- to milli-second time-scales in recombinant rMUP in both its ligand-free APO form and its HOLO form with the pheromone 2-methoxy-3-isobutylpyrazine. We believed that any differences in slow timescale dynamics may shed light on the hitherto unknown mechanism of ligand entry into the occluded bindingpocket of this protein.

\section{Materials and methods}

The transverse relaxation rates $R_{2}\left(1 / \tau_{\mathrm{cp}}\right)$ of ${ }^{15} \mathrm{~N}$ were measured as a function of the CPMG pulse repetition rate $1 / \tau_{\mathrm{cp}}$, where $\tau_{\mathrm{cp}}$ is the interval between two consecutive $\pi$ pulses, at a static magnetic field of $14.1 \mathrm{~T}$ (600 MHz for protons) at temperatures of $298 \mathrm{~K}$ and $308 \mathrm{~K}$. In this study we used relaxation-compensated CPMG sequences (Loria et al. 1999), which take into account the different relaxation rates of in- and antiphase single-quantum coherences $\mathrm{N}_{x}$ and $2 \mathrm{~N}_{x} \mathrm{H}_{z}$. We made simple modifications of the sequences $\mathrm{A}$ and $\mathrm{B}$ of Palmer and co-workers (Millet et al. 2000). The main modification, which is useful for proteins that are not only enriched in ${ }^{15} \mathrm{~N}$ but also in ${ }^{13} \mathrm{C}$, consists in the inclusion of $\pi$ pulses applied to both ${ }^{13} \mathrm{C}^{\prime}$ and ${ }^{13} \mathrm{C}^{\alpha}$ in the middle of the $\tau$ delays (i.e., at $1 / 4$ and $3 / 4$ of each interval $\tau_{\mathrm{cp}}=2 \tau$ between two consecutive ${ }^{15} \mathrm{~N}$ refocusing pulses) to attenuate the build-up of anti-phase terms $2 \mathrm{~N}_{x} \mathrm{C}_{z}{ }^{\prime}$ and $2 \mathrm{~N}_{x} \mathrm{C}_{z}^{\alpha}$ due to scalar couplings $\mathrm{J}\left({ }^{15} \mathrm{~N}^{13} \mathrm{C}^{\prime}\right) \approx 15 \mathrm{~Hz}$ and $\mathrm{J}\left({ }^{15} \mathrm{~N}^{13} \mathrm{C}^{\alpha}\right) \approx 4-10 \mathrm{~Hz}$. These anti-phase terms might otherwise become a source of errors for delays $\tau_{\mathrm{cp}}$ longer than a few milliseconds. Data were collected for pulse intervals $\tau_{\mathrm{cp}}=1.0,1.5$, $2.0,4.0,6.6,10.8$ and $21.6 \mathrm{~ms}$, corresponding to $1 / \tau_{\mathrm{cp}}=1000,667,500,250,152,93$ and $46 \mathrm{~Hz}$. For each $\mathrm{t}_{1}$ increment in the $2 \mathrm{D}$ experiments, 32 scans were accumulated for fast repetition rates $1 / \tau_{\mathrm{cp}} \geq 250 \mathrm{~Hz}$ while 64 scans were necessary to obtain a sufficient signal-to-noise ratio for experiments with $1 / \tau_{\mathrm{cp}}<$ $250 \mathrm{~Hz}$. Data processing has been performed using the GNU Package NMRPipe/NMRDraw/NlinLS (Delaglio et al. 1995). Relaxation rates were obtained by least-squares fitting of the decays to mono-exponential functions (Matlab 1992, Matlab Reference Guide, Natick, Massachusetts). Errors of the decay rates were estimated by a Monte-Carlo analysis using 200 synthetic data points. All experiments on APO- and HOLO-rMUP have been repeated twice. The corresponding data can be found in Supplementary Material, tables S1-S4. Relaxation dispersion plots have been interpolated using either the general formula of Eq. 1, or the simplified formula of Eq. 2 applicable for skewed populations, i.e., for $p_{\mathrm{a}} » p_{\mathrm{b}}$ (Ishima and Torchia 1999). Errors in the fitted parameters were again estimated using a Monte-Carlo analysis using 200 synthetic data points. Carver and Richards derived a general equation, which was later the subject of an erratum (Carver and Richards 1972), and then cast in a more convenient form (Davis et al. 1994; Jen 1978):

$$
\begin{aligned}
R_{2}\left(1 / \tau_{\mathrm{cp}}\right)= & \frac{1}{2}\left(R_{a}+R_{b}+k_{\mathrm{ex}}-\frac{1}{\tau_{\mathrm{cp}}}\right. \\
& \left.\times \cosh ^{-1}\left[D_{+} \cosh \left(\eta_{+}\right)-D_{-} \cos \left(\eta_{-}\right)\right]\right) .
\end{aligned}
$$

where $D_{ \pm}=1 / 2\left[ \pm 1+\left(\psi+2 \Delta \omega^{2}\right) /\left(\psi^{2}+\zeta^{2}\right)^{1 / 2}\right]$,

$$
\begin{gathered}
\eta_{ \pm}=\tau_{\mathrm{cp}} / \sqrt{2}\left[ \pm \psi+\left(\psi^{2}+\zeta^{2}\right)^{1 / 2}\right]^{1 / 2}, \\
\psi=\left(R_{a}-R_{b}-p_{\mathrm{a}} k_{\mathrm{ex}}+p_{\mathrm{b}} k_{\mathrm{ex}}\right)^{2}-\Delta \omega^{2}+4 p_{\mathrm{a}} p_{\mathrm{b}} k_{\mathrm{ex}}^{2}
\end{gathered}
$$

and $\zeta=2 \Delta \omega\left(R_{a}-R_{b}-p_{\mathrm{a}} k_{\mathrm{ex}}+p_{\mathrm{b}} k_{\mathrm{ex}}\right)$, other symbols having their usual meanings, i.e., $a$ and $b$ refer to the two conformations, $p_{i}$ to the populations, $R_{i}$ is the transverse relaxation rate of the $i^{\text {th }}$ conformation in 
Table 1 Exchange parameters for APO-rMUP at 308 and $298 \mathrm{~K}$, obtained by fitting the relaxation dispersion of the rates $R_{2}\left(1 / \tau_{\mathrm{cp}}\right)$ to Eq. 1 , unless indicated by a superscript a

NR; Residue not retained in the analysis

${ }^{a}$ Parameters that were obtained by fitting the relaxation dispersion data to Eq. 2 rather than Eq. 1

\begin{tabular}{|c|c|c|c|c|}
\hline Residue & $T(\mathrm{~K})$ & $p_{\mathrm{a}}$ & $k_{\mathrm{ex}}\left(\mathrm{s}^{-1}\right)$ & $\Delta \omega\left(\mathrm{s}^{-1}\right)$ \\
\hline \multirow[t]{2}{*}{ Ser 4} & 308 & NR & NR & NR \\
\hline & $298^{a}$ & $0.80 \pm 0.06$ & $161 \pm 90$ & $175 \pm 50$ \\
\hline \multirow[t]{2}{*}{ Glu 18} & 308 & $0.78 \pm 0.05$ & $1341 \pm 68$ & $157 \pm 6$ \\
\hline & 298 & $0.86 \pm 0.15$ & $436 \pm 31$ & $136 \pm 6$ \\
\hline \multirow[t]{2}{*}{ Arg 39} & 308 & $0.50 \pm 0.01$ & $1191 \pm 10$ & $184 \pm 1$ \\
\hline & 298 & $0.92 \pm 0.01$ & $197 \pm 27$ & $165 \pm 1$ \\
\hline \multirow[t]{2}{*}{ Leu 40} & 308 & $0.50 \pm 0.15$ & $1661 \pm 105$ & $275 \pm 10$ \\
\hline & 298 & $0.80 \pm 0.03$ & $800 \pm 31$ & $257 \pm 18$ \\
\hline \multirow{2}{*}{ Phe 41} & 308 & NR & NR & NR \\
\hline & $298^{\mathrm{a}}$ & $0.89 \pm 0.02$ & $48 \pm 20$ & $296 \pm 150$ \\
\hline \multirow[t]{2}{*}{ Thr 58} & 308 & $0.65 \pm 0.02$ & $524 \pm 39$ & $100 \pm 3$ \\
\hline & 298 & $0.50 \pm 0.01$ & $275 \pm 29$ & $93 \pm 2$ \\
\hline \multirow[t]{2}{*}{ Ser 68} & 308 & NR & NR & NR \\
\hline & 298 & $0.74 \pm 0.03$ & $152 \pm 26$ & $100 \pm 72$ \\
\hline \multirow[t]{2}{*}{ Met 69} & $308^{a}$ & $0.71 \pm 0.06$ & $626 \pm 150$ & $134 \pm 13$ \\
\hline & 298 & NR & NR & NR \\
\hline \multirow[t]{2}{*}{ Val 70} & 308 & $0.50 \pm 0.01$ & $675 \pm 16$ & $161 \pm 2$ \\
\hline & 298 & $0.76 \pm 0.01$ & $335 \pm 11$ & $141 \pm 2$ \\
\hline \multirow[t]{2}{*}{ Ala 71} & 308 & $0.72 \pm 0.06$ & $889 \pm 27$ & $163 \pm 5$ \\
\hline & 298 & NR & NR & NR \\
\hline \multirow[t]{2}{*}{ Asp 72} & 308 & $0.50 \pm 0.01$ & $1151 \pm 19$ & $241 \pm 3$ \\
\hline & 298 & $0.76 \pm 0.01$ & $571 \pm 32$ & $221 \pm 3$ \\
\hline \multirow[t]{2}{*}{ Glu 75} & 308 & NR & NR & NR \\
\hline & $298^{a}$ & $0.91 \pm 0.13$ & $284 \pm 20$ & $130 \pm 54$ \\
\hline \multirow[t]{2}{*}{ Ser 81} & 308 & $0.50 \pm 0.01$ & $1475 \pm 25$ & $343 \pm 4$ \\
\hline & 298 & $0.76 \pm 0.02$ & $823 \pm 50$ & $324 \pm 4$ \\
\hline \multirow[t]{2}{*}{ Val 82} & $308^{\mathrm{a}}$ & $0.93 \pm 0.13$ & $633 \pm 350$ & $703 \pm 190$ \\
\hline & 298 & NR & $\mathrm{NR}$ & NR \\
\hline \multirow[t]{2}{*}{ Thr 89} & 308 & $0.50 \pm 0.01$ & $1076 \pm 55$ & $377 \pm 11$ \\
\hline & 298 & $0.75 \pm 0.03$ & $884 \pm 68$ & $359 \pm 10$ \\
\hline \multirow[t]{2}{*}{ Asp 98} & 308 & $0.99 \pm 0.01$ & $409 \pm 100$ & $1389 \pm 360$ \\
\hline & 298 & NR & NR & NR \\
\hline \multirow[t]{2}{*}{ His 104} & 308 & NR & NR & NR \\
\hline & 298 & $0.50 \pm 0.05$ & $318 \pm 23$ & $103 \pm 2$ \\
\hline \multirow[t]{2}{*}{ Leu 105} & 308 & $0.79 \pm 0.28$ & $2040 \pm 343$ & $333 \pm 12$ \\
\hline & 298 & NR & NR & NR \\
\hline \multirow[t]{2}{*}{ Phe 114} & 308 & $0.50 \pm 0.01$ & $1375 \pm 44$ & $165 \pm 4$ \\
\hline & 298 & $0.91 \pm 0.01$ & $114 \pm 22$ & $145 \pm 3$ \\
\hline \multirow[t]{2}{*}{ Leu 116} & 308 & $0.50 \pm 0.01$ & $1965 \pm 372$ & $198 \pm 30$ \\
\hline & 298 & $0.73 \pm 0.06$ & $35 \pm 4$ & $178 \pm 30$ \\
\hline \multirow[t]{2}{*}{ Gly 118} & 308 & $0.90 \pm 0.06$ & $519 \pm 400$ & $99 \pm 24$ \\
\hline & 298 & $0.63 \pm 0.20$ & $20 \pm 12$ & $113 \pm 21$ \\
\hline
\end{tabular}

the absence of chemical exchange, $\Delta \omega$ is the difference between the chemical shifts of the two conformations and $k_{\mathrm{ex}}=k_{\mathrm{ab}}+k_{\mathrm{ba}}$ is the exchange rate constant. Ishima and Torchia have proposed a simplified equation:

$$
\begin{array}{r}
R_{2}\left(1 / \tau_{\mathrm{cp}}\right)=R_{2}\left(1 / \tau_{\mathrm{cp}} \rightarrow \infty\right)+p_{\mathrm{a}} p_{\mathrm{b}} \Delta \omega^{2} k_{\mathrm{ex}} / \\
{\left[k_{\mathrm{ex}}^{2}+\left(p_{\mathrm{a}}^{2} \Delta \omega^{4}+144 / \tau_{\mathrm{cp}}^{4}\right)^{1 / 2}\right]}
\end{array}
$$

which is only applicable if the populations are very unequal, i.e., if $p_{\mathrm{a}} \gg p_{\mathrm{b}}$ (Ishima and Torchia 1999).

A recent procedure uses the fitting of all residues with a common set of kinetic parameters $\left(p_{\mathrm{a}}\right.$ and $\left.k_{\mathrm{ex}}\right)$, in order to reveal the presence of collective motions (Beach et al. 2005; Mulder et al. 2001b). This analysis has been performed on our data, and the results suggest that there are no collective motions. Thus the parameters reported in Table 1 result from individual fitting.

It should be noted that pheromone complexation affects the correlation times $\left(\tau_{\mathrm{c}}=7.4\right.$ and $7.1 \mathrm{~ns}$ for APO-rMUP at 298 and $308 \mathrm{~K}$, respectively, and $\tau_{\mathrm{c}}=9.2$ and $8.9 \mathrm{~ns}$ for HOLO-rMUP at 298 and no incidence on internal motions as determined by CPMG dispersion of relaxation rates.

\section{Results and discussion}

In the APO form at $308 \mathrm{~K}$, out of a total of 162 amino acids in rMUP, 92 residues were retained for the analysis, i.e., the residues that do not suffer from $308 \mathrm{~K})$. This effect, which has yet to be explained, has 
Fig. 1 Relaxation dispersion plots for APO- (cyan squares) and HOLO-rMUP (red diamonds) at $308 \mathrm{~K}$ for Arg 39 and Asp 72. For APOrMUP, the curve results from fitting to Eq. 1. The exchange rates are given in Table 1 . For HOLO-rMUP, the curve represents the average of the rates over $1 / \tau_{\mathrm{cp}}$
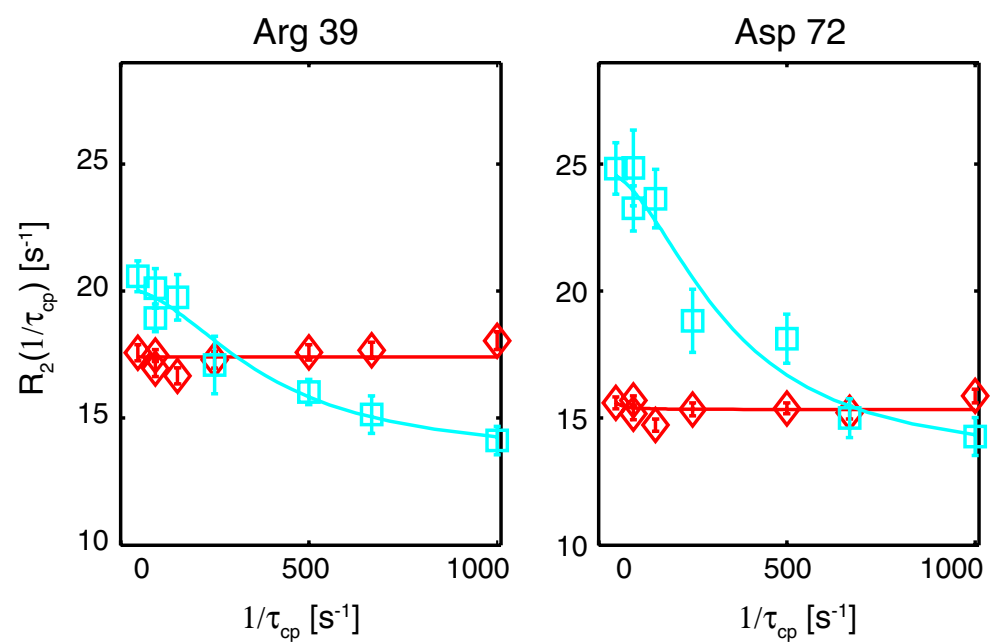

spectral overlap or low signal-to-noise ratios. Among the residues that could be analysed, up to 16 show detectable presence of chemical exchange, in the sense that $R_{2}$ depends on $1 / \tau_{\mathrm{cp}}$. These residues are Glu 18, Arg 39, Leu 40, Thr 58, Met 69, Val 70, Ala 71, Asp 72, Ser 81, Val 82, Thr 89, Asp 98, Leu 105, Phe 114, Leu 116 and Gly 118. The exchange parameters obtained by fitting to Eqs. 1 or 2 are reported in Table 1. In the HOLO form of rMUP bound to the pheromone IBMP, at the same temperature of $308 \mathrm{~K}, 107$ residues could be retained for the analysis, using the same criteria for discarding residues as in the APO form. None of these residues show any evidence for chemical exchange, since $R_{2}$ does not depend on $1 / \tau_{\mathrm{cp}}$ in the HOLO form. Fig. 1 shows a comparison of $R_{2}\left(1 / \tau_{\mathrm{cp}}\right)$ for two typical residues Arg 39 and Asp 72. The absence of any chemical exchange in the HOLO form and its presence in the APO form is clearly seen.

As discussed elsewhere (Millet et al. 2000; Mulder et al. 2001a, b; McElheny et al. 2005), chemical exchange processes cannot be fully characterized by a single set of experiments at a single magnetic field and a single temperature. We could not investigate the field-dependence, but we have repeated the experiments at a lower temperature of $298 \mathrm{~K}$ to At this temperature, it was possible to retain 96 residues for the analysis in the HOLO complex and 92 for the APO form. As many as 18 of the latter show the presence of chemical exchange. No less than 13 of these 18 residues were also found to be affected by chemical exchange at $308 \mathrm{~K}$. The differences in the numbers of residues that could be characterised at the two temperatures result from different intensities in the cross-peaks which can lead to large errors in the observed rates $R_{2}\left(1 / \tau_{\mathrm{cp}}\right)$, or from spectral overlap at one of the two temperatures. In the APO form, the relaxation dispersion curves $R_{2}\left(1 / \tau_{\mathrm{cp}}\right)$ of two representative residues at the two temperatures are shown in Fig. 2. As expected, the values of the exchange rate constant, $k_{\mathrm{ex}}$, decrease with the temperature, see Table 1. Once again, the HOLO form of rMUP does not show any evidence of chemical exchange, in agreement with the behaviour at $308 \mathrm{~K}$. It was not possible to increase the temperature beyond $308 \mathrm{~K}$ because of the limited stability of the protein. Nevertheless, we expect motions in the HOLO form connected with the release of the pheromone. A decrease in the temperature usually slows down exchange processes; if they are too fast to be brought to light on the CPMG time scale at a higher temperature, they might fall within the range of detectability of this technique at a lower temperature.

Kinetic theory predicts a decrease in exchange rates at lower temperatures, since the conformational exchange processes are slowed down. The temperature dependence of $k_{\text {ex }}$ can be described by the Arrhenius equation, $k_{\mathrm{ex}}=A_{0} \exp \left\{-E_{a} /\left(k_{\mathrm{B}} T\right)\right\} /$ $\left(1-p_{\mathrm{a}}\right)$ and by the Boltzmann distribution $p_{\mathrm{a}}=(1+$ $\left.\exp \left\{-\Delta G / k_{\mathrm{B}} T\right\}\right)^{-1}$, where $A_{0}$ is a pre-exponential factor, $E_{a}$ the activation energy of the process, $k_{\mathrm{B}}$ the Boltzmann constant and $\Delta G$ the difference in free energy between the two states $a$ and $b$ (Mandel et al. 1996). To a first approximation the difference in chemical shifts $\Delta \omega$ is considered to remain constant regardless of temperature (Palmer et al. 2001), so that the ratio $k_{\mathrm{ex}} / \Delta \omega$ shifts to lower values with decreasing temperature. The $R_{\text {ex }}$ values have been computed using Eq. 3 (Luz and Meiboom 1963), which, under the conditions of our experiments (Mandel et al. 1996), provides an approximation to within $5 \%$ of the exact formulation (Allerhand and Gutowsky 1965). 
Fig. 2 Relaxation dispersion plots for APO-rMUP at $308 \mathrm{~K}$ (cyan squares) and $298 \mathrm{~K}$ (blue circles) for Arg 39 and for Val 70. The curves were obtained by fitting to Eq. 1 . The exchange rates are given in Table 1
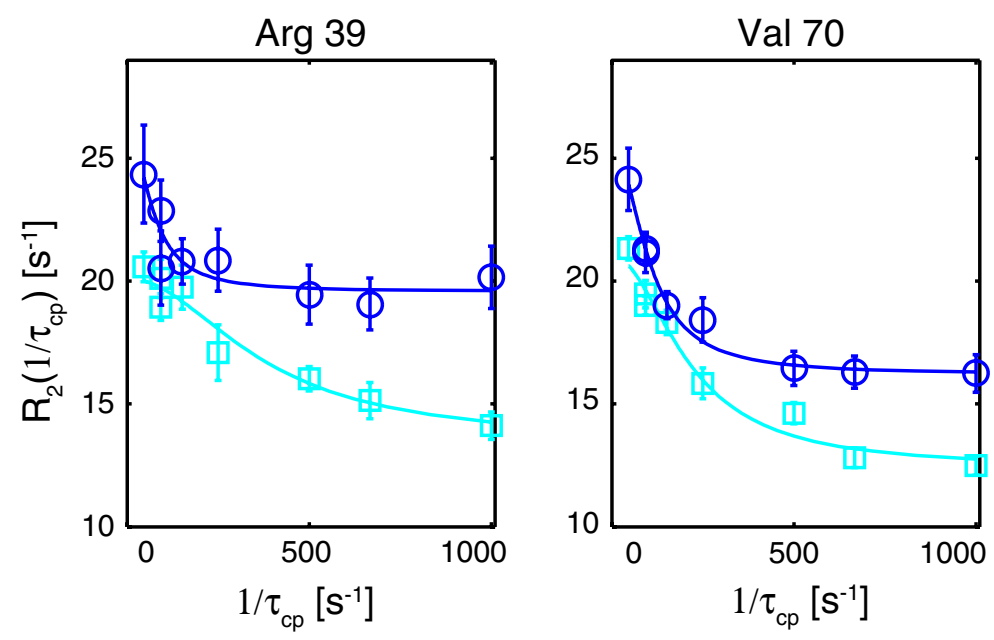

$R_{\mathrm{ex}}=\frac{p_{\mathrm{a}} p_{\mathrm{b}} \Delta \omega^{2}}{k_{\mathrm{ex}}}\left[1-\frac{2}{k_{\mathrm{ex}} \tau_{\mathrm{cp}}} \tanh \left(\frac{k_{\mathrm{ex}} \tau_{\mathrm{cp}}}{2}\right)\right]$

Using Eq. 3 for a fixed value of $\tau_{\mathrm{cp}}=1.2 \mathrm{~ms}$, Mandel demonstrated that the exchange rate $R_{\text {ex }}$ reaches a maximum when $k_{\mathrm{ex}}=3.2 / \tau_{\mathrm{cp}}$. For fast exchange $k_{\mathrm{ex}} \gg 3.2 / \tau_{\mathrm{cp}}, R_{\mathrm{ex}}=p_{\mathrm{a}} p_{\mathrm{b}} \Delta \omega^{2} / k_{\mathrm{ex}}$ and for slow exchange $k_{\mathrm{ex}} \ll 3.2 / \tau_{\mathrm{cp}}, R_{\mathrm{ex}}$ tends to zero (Mandel et al. 1996). As seen previously, an increase in the temperature leads to an increase of $k_{\mathrm{ex}}$, so that augmenting $T$ leads to a decrease of $R_{\mathrm{ex}}$ if $k_{\mathrm{ex}}>3.2 / \tau_{\mathrm{cp}}$, and to an increase of $R_{\mathrm{ex}}$ if $k_{\mathrm{ex}}<3.2 / \tau_{\mathrm{cp}}$. As a consequence, the sign of the derivative $\mathrm{d} R_{\mathrm{ex}} / \mathrm{d} T$ indicates whether the exchange rate is faster or slower than $k_{\mathrm{ex}}=3.2 / \tau_{\mathrm{cp}}$. The temperature dependence of $R_{\mathrm{ex}}$ is reported in Table S5. All of the 13 residues that can be observed in APO-

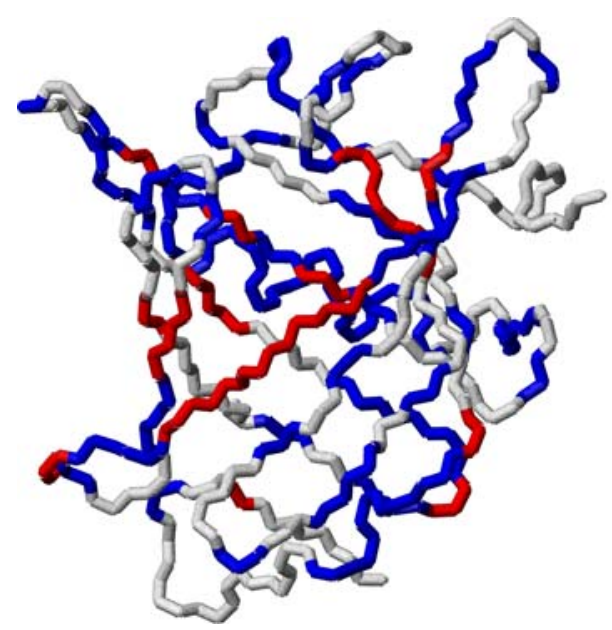

Fig. 3 Stereo view of APO-rMUP where residues featuring chemical exchange have been coloured in red, while residues for which relaxation rates could be recorded (at either temperature)
rMUP at both temperatures have positive derivatives $\mathrm{d} R_{\mathrm{ex}} / \mathrm{d} T$, indicating that the exchange rate is slower than $k_{\mathrm{ex}}=3.2 / \tau_{\mathrm{cp}}$.

A total of 21 residues in APO-rMUP show evidence of chemical exchange at least at one of the two temperatures $(T=298$ or $308 \mathrm{~K})$. Most of these residues are located in the $\beta$-barrel (see Fig. 3), while 5 residues lie in loops, i.e., in regions where one expects motions. It is interesting to note that the residues from Ser 68 to Asp 72, as well as Ser 81 and Val 82, are spatially close, and constitute the last part of the $\beta \mathrm{D}$ sheet as well as the centre of the following $\beta \mathrm{E}$ sheet. Although residues that constitute the $\alpha$-helix are well represented (79\% of the residues in the helix are detectable, see Supplementary Material), none of them shows any evidence of chemical exchange. Fig. 3 shows residues for which

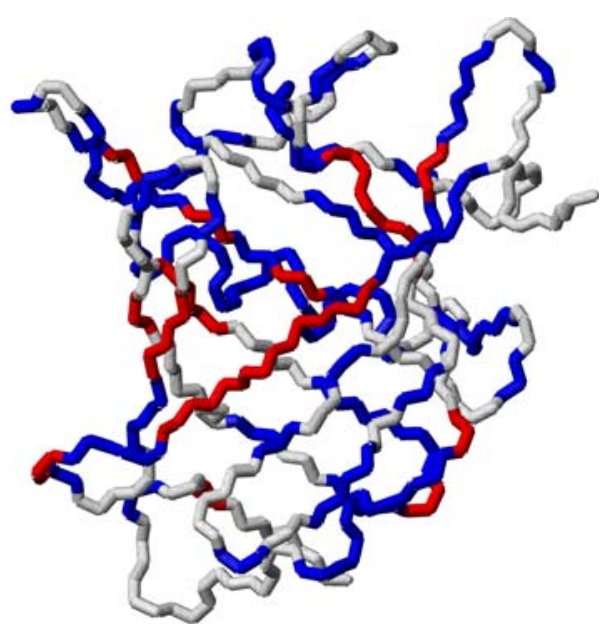

are coloured in blue. Residues for which relaxation data are unavailable at either temperature are coloured in grey 
relaxation data could be measured (at either of the two temperatures) coloured in blue, with residues featuring chemical exchange (at either of the two temperatures) coloured in red.

It is clear from Fig. 3 that residues exhibiting conformational exchange are located principally adjacent to the $\beta$-turn comprising residues $83-87$. To the best of our knowledge, the mechanism of entry of a ligand into the occluded binding pocket of APO-rMUP has not been reported. However, in other members of the lipocalin family, it has been suggested that slow timescale conformational changes in the APO form are related to ligand entry. For example, Rüterjans and coworkers (Franzoni et al. 2002) report increased backbone disorder in the $\beta \mathrm{E}-\beta \mathrm{F}$ turn in NMR structure calculations of retinol binding protein, which correlates with a suggested portal for pheromone entry. Similarly, Zhang et al. have reported disorder in the same region of human intestinal fatty acid binding protein (Zhang et al. 1997). This region is analogous to residues 83-87 in rMUP, and suggests that the ligand gains entry through an equivalent portal. However, the lack of any obvious correlation between either exchange rates or populations of 'excited' states of residues listed in Table 1 suggests that there is no concerted largescale conformational rearrangement that facilitates ligand entry. Instead, it is likely that the ligand gains entry via stochastic 'breathing' motions of the protein.

The dramatic and complete loss of conformational exchange in rMUP upon pheromone binding stands in stark contrast with a recent study (based on spectral density mapping) where no significant differences in slow internal motions on the $\mu$ s-ms time-scale were noted between APO and HOLO forms (Krížová et al. 2004). It is not possible to determine with certainty the reasons for this discrepancy from the present study, but the absence of differences in slow internal motions between APO and HOLO forms in that study might be related to the presence of some unknown endogenous ligand in the APO form. In early work we noted that over-expressed recombinant MUP contains bound endogenous ligands from the expression medium, and we routinely use an ethanol precipitation step during purification to remove such ligands. MUP is a promiscuous binder of small hydrophobic molecules, and indeed in our hands it is critical that the protein is only handled and stored in glass, since it readily binds trace amounts of plasticizer from polypropylene tubes for example. The presence of endogenous ligands manifests itself in significant chemical-shift changes of binding-site residues in ${ }^{15} \mathrm{~N}-{ }^{1} \mathrm{H}$ HSQC spectra in comparison with the true APO (ethanol-precipitated) protein. Previous NMR and thermodynamic studies on
MUP do not report the use of an ethanol precipitation step (Abbate et al. 1999; Franzoni et al. 2002; Lücke et al. 1999, Zídek et al. 1999a, b). The backbone shift assignments that we obtain for the ethanol-precipitated APO protein differ in the binding-site region from those originally reported by Rüterjans and co-workers (Abbate et al. 1999; Lücke et al. 1999). These observations cannot however explain the presence of slow time-scale motions in the HOLO form of the protein in the study of Krížová compared with a complete loss of such motions in the present study. This discrepancy may be related to the influence of different ligands on slow time-scale dynamics in the respective complexes.

Acknowledgements $\mathrm{We}$ are indebted to Dr Julien Wist (Bogotá, Columbia) for stimulating discussions. This work was supported by the Swiss National Science Foundation, the Swiss Commission for Innovation and Technology, the French CNRS, and the BBSRC (grant no. B19388 to SWH, United Kingdom).

\section{References}

Abbate F, Franzoni L, Löhr F, Lücke C, Ferrari E, Sorbi RT, Rüterjans H, Spisni A (1999) Letter to the editor: complete ${ }^{1} \mathrm{H},{ }^{15} \mathrm{~N}$ and ${ }^{13} \mathrm{C}$ assignment of a recombinant mouse major urinary protein. J Biomol NMR 15:187-188

Akke M (2002) NMR methods for characterizing microsecond to millisecond dynamics in recognition and catalysis. Curr Opin in Struc Biol 12:642-647

Allerhand A, Gutowsky HS (1965) Spin-echo studies of chemical exchange. II. Closed formulas for two sites. J Chem Phys 42:1587-1599

Beach H, Cole R, Gill ML, Loria JP (2005) Conservation of $\mu \mathrm{s}-$ ms enzyme motions in the apo- and substrate-mimicked state. J Am Chem Soc 127:9167-9176

Bingham RJ, Findlay JBC, Hsieh SY, Kalverda AP, Kjellberg A, Perazzolo C, Phillips SEV, Seshadri K, Trinh $\mathrm{CH}$, Turnbull WB, Bodenhausen G, Homans SW (2004) Thermodynamics of binding of 2-methoxy-3-isopropylpyrazine and 2-methoxy-3-isobutylpyrazine to the major urinary protein. J Am Chem Soc 126:1675-1681

Carr HY, Purcell EM (1954) Effects of diffusion on free precession in NMR experiments. Phys Rev 94:630-638

Carver JP, Richards RE (1972) A general two-site solution for the chemical exchange produced dependence of T2 upon Carr-Purcell pulse separation. J Magn Reson 6:89-105; erratum (1972) J Magn Reson 8:206

Davis DG, Perlman ME, London RE (1994) Direct measurements of the dissociation-rate constant for inhibitor-enzyme complexes via the $\mathrm{T}_{1}$ rho and $\mathrm{T}_{2}$ (CPMG) methods. J Magn Reson Ser B 104:266-275

Delaglio F, Grzesiek S, Vuister GW, Zhu G, Pfeifer J, Bax A (1995) NMRPipe: A multidimensional spectral processing system based on UNIX pipes. J Biomol NMR 6:277-293

Deverell C, Morgan RE, Strange JH (1970) Studies of chemical exchange by nuclear magnetic relaxation in the rotating frame. Mol Phys 18:553-559

Ferrari E, Lodi T, Sorbi RT, Tirindelli R, Cavaggioni A, Spisni A (1997) Expression of a lipocalin in Pichia pastoris: secretion, 
purification and binding activity of a recombinant mouse major urinary protein. FEBS Letters 401:73-77

Franzoni L, Lücke C, Pérez C, Cavazzini D, Rademacher M, Ludwig C, Spisni A, Rossi GL, Rüterjans H (2002) Structure and backbone dynamics of apo- and holo-cellular retinolbinding protein in solution. J Biol Chem 277:21983-21997

Frueh D (2002) Internal motions in proteins and interference effects in nuclear magnetic resonance. Prog NMR Spectrosc 41:305-324

Früh D, Chiarparin E, Pelupessy P, Bodenhausen G (2002) Measurement of long-range cross-correlation rates using a combination of single- and multiple-quantum NMR spectroscopy in one experiment. J Am Chem Soc 124:4050-4057

Ishima R, Torchia DA (1999) Estimating the time scale of chemical exchange of proteins from measurements of transverse relaxation rates in solution. $\mathrm{J}$ Biomol NMR 14:369-372

Ishima R, Torchia DA (2005) Error estimation and global fitting in transverse-relaxation dispersion experiments to determine chemical-exchange parameters. J Biomol NMR 32:4154

Jen J (1978) Chemical exchange and NMR $T_{2}$ relaxation-the multisite case study. J Magn Reson 30:111-128

Kloiber K, Konrat R (2000) Differential multiple-quantum relaxation arising from cross-correlated time-modulation of isotropic chemical shifts. J Biomol NMR 18:33-42

Krížová H, Zídek L, Stone MJ, Novotny MV, Sklenáŕ V (2004) Temperature-dependent spectral density analysis applied to monitoring backbone dynamics of major urinary protein-I complexed with the pheromone 2-sec-butyl-4,5dihydrothiazole. J Biomol NMR 28:369-384

Lipari G, Szabo A (1982a) Model-free approach to the interpretation of nuclear magnetic resonance relaxation in macromolecules. 1. Theory and range of validity. J Am Chem Soc 104:4546-4559

Lipari G, Szabo A (1982b) Model-free approach to the interpretation of nuclear magnetic resonance relaxation in macromolecules. 2. Analysis of experimental results. J Am Chem Soc 104:4559-4570

Loria JP, Rance M, Palmer III AG (1999) A relaxationcompensated Carr-Purcell-Meiboom-Gill sequence for characterizing chemical exchange by NMR spectroscopy. J Am Chem Soc 121:2331-2332

Lücke C, Franzoni L, Abbate F, Löhr F, Ferrari E, Sorbi RT, Rüterjans H, Spisni A (1999) Solution structure of a recombinant mouse Major Urinary Protein. Eur J Biochemistry $266: 1210-1218$

Luz Z, Meiboom S (1963) Chemical exchange and NMR-T relaxation. J Chem Phys 39:366-370

Mandel AM, Akke M, Palmer III AG (1996) Dynamics of ribonuclease $\mathrm{H}$ : temperature dependence of motions on multiple time scales. Biochemistry 35:16009-16023
McElheny D, Schnell JR, Lansing JC, Dyson HJ, Wright PE (2005) Defining the role of active-site loop fluctuations in dihydrofolate reductase catalysis. Proc Nat Acad Sci USA 102:5032-5037

Meiboom S, Gill D (1958) Modified spin-echo method for measuring nuclear relaxation times. Rev Scient Instr 29:688691

Millet O, Loria JP, Kroenke CD, Pons M, Palmer III AG (2000) The static magnetic field dependence of chemical exchange linebroadening defines the NMR chemical shift time scale. J Am Chem Soc 122:2867-2877

Mulder FAA, Skrynnikov NR, Hon B, Dahlquist FW, Kay LE (2001a) Measurement of slow ( $\mu$ s-ms) time scale dynamics in protein side chains by ${ }^{15} \mathrm{~N}$ relaxation dispersion NMR spectroscopy: application to Asn and Gln residues in a cavity mutant of T4 lysozyme. J Am Chem Soc 123:967-935

Mulder FAA, Mittermaier A, Hon B, Dahlquist FW, Kay LE (2001b) Studying excited states of proteins by NMR spectroscopy. Nature Struc Biol 8:932-935

Mulder FAA, Hon B, Mittermaier A, Dahlquist FW, Kay LE (2001c) Slow internal dynamics in proteins: application of NMR relaxation dispersion spectroscopy to methyl groups in a cavity mutant of T4 lysozyme. J Am Chem Soc 124:1443-1451

Palmer III AG, Kroenke CD, Loria JP (2001) Nuclear magnetic resonance methods for quantifying microsecond-to-millisecond motions in biological macromolecules. In: Methods in Enzymology, vol 339 Academic Press, p 204

Wagner G, Bodenhausen G, Müller N, Rance M, Sørensen OW, Ernst RR, Wüthrich K (1985) Exchange of two-spin order in nuclear magnetic resonance: separation of exchange and cross-relaxation processes. J Am Chem Soc 107:6440-6446

Wang C, Palmer III AG (2003) Solution NMR methods for quantitative identification of chemical exchange in ${ }^{15} \mathrm{~N}$ labeled proteins. Magn Reson Chem 41:866-876

Wist J, Frueh D, Tolman JR, Bodenhausen G (2004) Triple quantum decoherence under multiple refocusing: slow correlated chemical shift modulations of $\mathrm{C}^{\prime}$ and $\mathrm{N}$ nuclei in proteins. J Biomol NMR 28:263-272

Zhang F, Lücke C, Baier LJ, Sacchettini JC, Hamilton JA (1997) Solution structure of human intestinal fatty acid binding protein: implications for ligand entry and exit. J Biomol NMR 9:213-228

Zídek L, Novotny MV, Stone MJ (1999a) Increased protein backbone conformational entropy upon hydrophobic ligand binding. Nat Struct Mol Biol 6:1118-1121

Zídek L, Stone MJ, Lato SM, Pagel MD, Miao Z, Ellington AD, Novotny MV (1999b) NMR mapping of the recombinant mouse major urinary protein I binding site occupied by the pheromone 2-sec-butyl-4,5-dihydrothiazole. Biochemistry 38:9850-9861 